\title{
Compressed Sensing Reconstruction of Smooth Signals in Electron Tomography
}

\author{
Rowan Leary ${ }^{1}$, Zineb Saghi ${ }^{1}$, Paul A. Midgley ${ }^{1}$ and Daniel J. Holland ${ }^{2}$ \\ 1. Department of Materials Science and Metallurgy, University of Cambridge, Pembroke Street, \\ Cambridge, CB2 3QZ, UK. \\ 2. Department of Chemical Engineering and Biotechnology, University of Cambridge, New Museums \\ Site, Pembroke Street, Cambridge CB2 3RA, UK.
}

Electron tomography (ET) has become an invaluable technique for the study of nanoscale systems, but the $3 \mathrm{D}$ reconstructions obtained using established algorithms may contain significant artifacts when the number or angular range of projections that can be recorded is limited. The fidelity of tomographic reconstructions from limited data can potentially be improved by incorporating some additional prior knowledge about the specimen, or 'regularization,' during the reconstruction process. The method of discrete tomography, for example, can be used to provide high-quality ET reconstructions when the specimen can be considered in discrete terms [1]. A significant challenge in ET is the faithful reconstruction of signals that show a smooth or piecewise smooth change in the property of interest (due to their very nature, such signals cannot be addressed using discrete algorithms).

Concepts of theoretical and practical signal recovery from undersampled data have recently undergone a paradigm shift with the development of a new sensing and recovery strategy known as compressed sensing (CS) [2,3]. CS theory shows that, under certain circumstances, exact signal recovery, or recovery with bounded discrepancy, may be possible from far fewer measurements than traditional theories dictate should be necessary. CS entails exploiting prior knowledge of transform sparsity or compressibility implicit in many signals (such as images), meaning that the signal can be represented or well-approximated, respectively, in a more compact form. Recovery is achieved through an optimization process that seeks the sparsest signal in the chosen transform domain that is consistent with the measured data. We have previously applied CS-ET in the study of uniform density ('piecewise constant') objects, showing the ability to obtain high-fidelity reconstructions, even from far fewer projections than are normally used [4]. Here we show that CS can enable high-fidelity reconstruction of smooth signals in ET.

There are now many powerful sparsifying transforms available. As shown by their success in the ubiquitous JPEG-2000 image compression algorithm, discrete wavelet transforms (DWTs), in particular, can provide sparse approximation of a range of signal content. DWTs can focus on localized structures with a zooming procedure that enables a so-called 'multiresolution' analysis, capturing both spatial position and spatial frequency information. Wavelets can therefore very effectively represent smooth and piecewise smooth signal content, including non-periodic point singularities such as jumps and spikes. Alternative sparsifying transforms that may be preferable for some particular forms of smooth signal content include, amongst others, the discrete cosine and curvelet transforms [5].

To demonstrate the efficacy of CS-ET, Figure 1a shows a test phantom containing smooth objects, of which simulated noisy projections have been generated. Figure $1 \mathrm{~b}$ shows a typical ET reconstruction from the projections, obtained using the conventional SIRT algorithm, which clearly suffers from artifacts arising from the limited sampling; in particular elongation of the objects in the 'missing-wedge' 
(vertical) direction and poor recovery of the intensity of the smaller objects. A reconstruction obtained using CS-ET on the other hand, in this case by promoting sparsity in a wavelet domain during reconstruction, is of much higher fidelity (Figure 1c). As shown in Figures 1d and e, while the SIRT reconstruction degrades substantially, robust CS-ET reconstruction is still possible using a very small number of well-chosen projections.

The ability to obtain high-fidelity reconstructions of smooth signals using CS-ET is significant as it offers the potential to substantially broaden the range of samples or phenomena that are amenable to study by ET. These include, for example, 3D analysis of compositional gradients or mapping of physical properties using CS-ET, a first example of which is the study of Nicoletti et al. [6]. Moreover, through use of an appropriate sparsifying transform, CS-ET is applicable to many other types of image content, promising the capability to obtain high-fidelity reconstructions of a broad range of samples across both the physical and life sciences [7].

\section{References}

[1] KJ Batenburg et al, Ultramicroscopy 109 (2009) p. 730.

[2] EJ Candès, J Romberg and T Tao, IEEE Trans. Inf. Theory 52 (2006) p. 489.

[3] DL Donoho, IEEE Trans. Inf. Theory 52 (2006) p. 1289.

[4] Z Saghi et al, Nano Lett. 11 (2011) p. 4666.

[5] JL Starck et al, "Sparse image and signal processing", (Cambridge University Press, Cambridge).

[6] O Nicoletti et al, (submitted).

[7] The research leading to these results has received funding from the European Research Council under the European Union's Seventh Framework Programme (FP7/2007-2013)/ERC grant agreement 291522-3DIMAGE. DJH acknowledges the support of the EPSRC (EP/F041772/1 and EP/F047991/1).
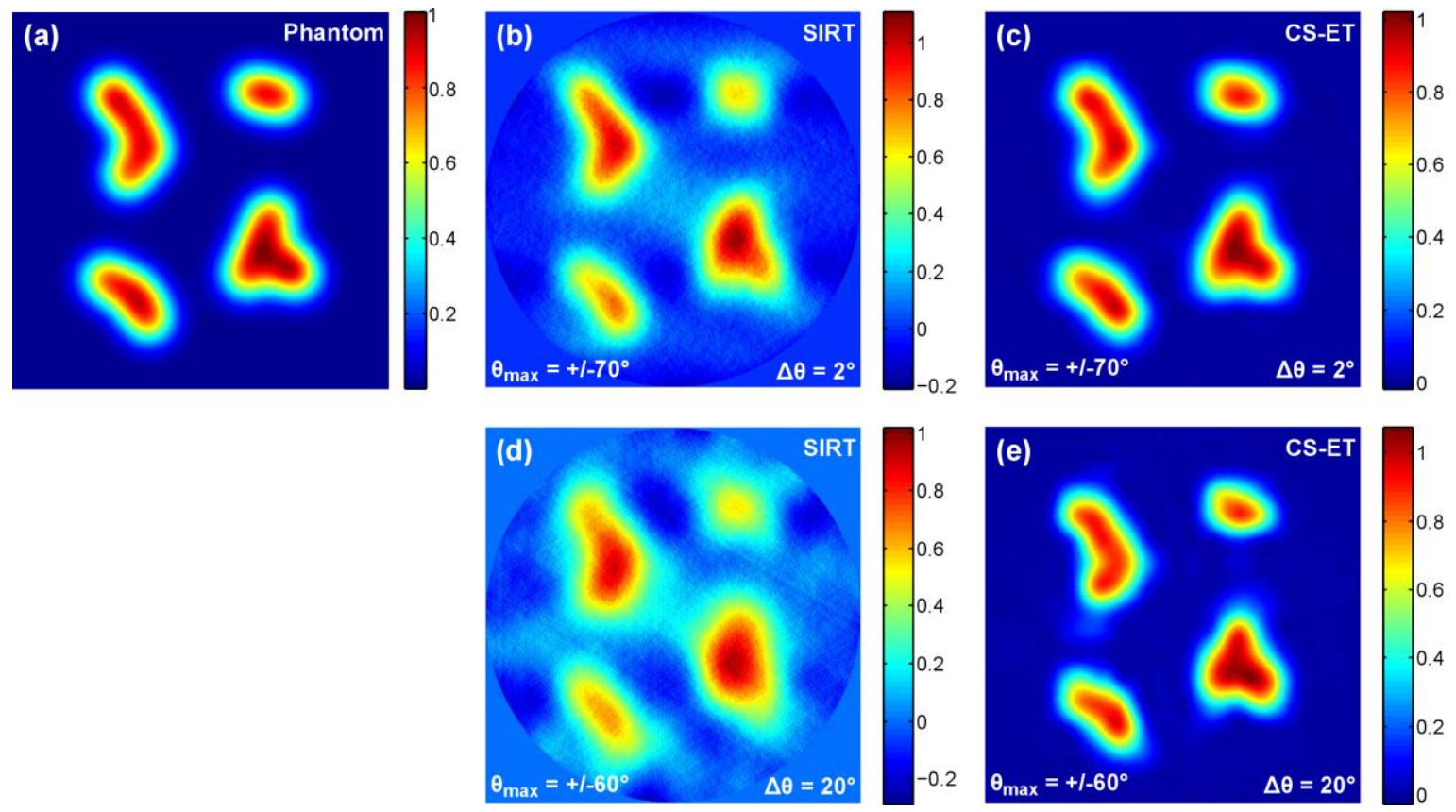

Figure 1. (a) Test phantom containing regions of smoothly decaying intensity, including anisotropic shapes, concavities and regions of differing size and intensity. (b,d) SIRT and (c,e) CS-ET reconstructions from simulated tilt-series projections of the phantom, with maximum tilt angle $\theta_{\max }$ and tilt increment $\Delta \theta$. 